\title{
NACIONALISMO Y DEMOCRACIA: REFLEJO DE AQUELLA RELACIÓN EN EL PRIMER AUTONOMISMO AZORIANO Y CANARIO
}

\author{
NATIONALISM AND DEMOCRACY: REFLECTION OF THAT \\ RELATIONSHIP IN THE FIRST AZOREAN AND CANARIAN \\ AUTONOMISM
}

Jorge Antonio Montesdeoca*

Fecha de Recepción: 14 de octubre de 2019

Fecha de Aceptación: 06 de mayo de 2020

Cómo citar este artículo/Citation: Jorge Antonio Montesdeoca (2021). Nacionalismo y democracia: reflejo de aquella relación en el primer autonomismo azoriano y canario. Anuario de Estudios Atlánticos; $n^{\circ}$ 67: 067-018.

http://anuariosatlanticos.casadecolon.com/index.php/aea/article/view/10589/10168

ISSN 2386-5571. https://doi.org/10.36980/10589.10168

Resumen: Nacionalismo y democracia son fenómenos políticos de una extraordinaria magnitud consolidados en el siglo pasado. Su interrelación ha interesado al mundo académico, que a menudo resalta aspectos como su contradicción, oposición o vinculación. Este artículo asume la perspectiva académica que señala una estrecha relación entre nacionalismo y alguno de los principios básicos de la democracia, sirviéndose de tal vinculación para analizar un estadio decisivo dentro del desarrollo político de dos archipiélagos atlánticos: Azores y Canarias. Con base en este enfoque novedoso se presentan las manifestaciones autonomistas acaecidas en ambas islas en el tránsito del siglo XIX al XX, ante todo, como un fenómeno nacionalista, ligado en el caso azoriano con la consolidación en las islas de una identidad nacional portuguesa $y$, en el caso canario, con el difícil tránsito del nacionalismo por Canarias durante el siglo XIX.

Palabras claves: nacionalismo, democracia, identidad nacional, autonomismo, Azores y Canarias

\begin{abstract}
Nationalism and Democracy are two political phenomena of an extraordinary magnitude firmly consolidated during the last century. Their interrelation has interested the academic world often highlighting its opposition, contradiction or linkage. This article assumes the academic perspective that indicates a close relationship between nationalism and some of the basic tenets of democracy. This connection will be useful in order to analyse a decisive stage in the political development of two Atlantic archipelagos: the Azores and the Canaries. Following this novel approach, the article shows the autonomist claims in both archipelagos during the years of transition from the XIX to the XX century, first and foremost as a nationalist phenomenon, linked in the Azores case to the consolidation in these islands of a Portuguese national identity, and in the case of the Canaries linked to the complicated contact of nationalism with the Canarian population thorough the XIX century.
\end{abstract}

Keywords: nationalism, democracy, national identity, autonomism, Azores and Canary Islands.

* Doctorando en el programa Islas Atlánticas: Historia, Patrimonio y Marco Jurídico Institucional. Departamento de Derecho Público. Universidad de Las Palmas de Gran Canaria. España. Correo electrónico: jorge.montesdeoca101@alu.ulpgc.es 


\section{VINCULACIÓN ENTRE NACIONALISMO Y DEMOCRACIA EN EL MUNDO ACADÉMICO}

Hoy en día el concepto de nacionalismo presenta una diversidad tan grande en usos y significado a nivel popular y cotidiano, que parece habérsele despojado del trascendental papel que ha desempeñado en el transcurso de la historia política y social de los últimos siglos. Actualmente entendido como un término oscuro y confuso, el mundo académico se presenta del todo aislado en su esfuerzo por descifrar la esencia y acepciones de aquel concepto y los fenómenos aparejados a aquel, así como en su afán por mesurar el alcance transformador que ha mostrado y continúa mostrando desde un plano histórico-político-social. El esfuerzo de tantos académicos por acercarse al estudio del nacionalismo desde un rigor científico parece eternamente quedar diluido ante una vorágine de acontecimientos, presuntamente relacionados con aquel, que a diario se reproducen en los medios de comunicación.

El presente trabajo propone alejarse de la indefinición y el obscurantismo que persiguen hoy en día el concepto de nacionalismo. Sobre la base de una producción científica desarrollada por reconocidos autores de aquel campo, se concebirá al nacionalismo como una variable válida y de gran interés al servicio de la historiografía y politología, con la cualidad a su vez de permitir abrir nuevas vías de entendimiento acerca del desarrollo sociopolítico ocurrido en determinados territorios. El artículo se centrará así en la vinculación entre nacionalismo y el concepto de democracia, en tanto que, según algunos académicos, la idea nacional se encuentra estrechamente relacionada con principios democráticos básicos.

La mayor parte de estudiosos del nacionalismo hacen una distinción entre un tipo cívico y otro étnico. El gran historiador Hobsbawm (1992), por ejemplo, a pesar de su crítica marxista del nacionalismo, defiende la aparición en primer lugar de una versión liberal del nacionalismo a comienzos del siglo XIX seguida de una versión étnica/romántica más reactiva que surgiría a finales del siglo XIX. Así, mientras unos autores fijan el concepto de nacionalismo centrándose más en sus aspectos étnicos, otros autores muestran mayor interés en sus aspectos cívicos (esto es, liberales y democráticos). El presente artículo basará su argumentación en este último grupo.

A este respecto, Nodia (1992, pp. 4-7) hace alusión a una especie de «matrimonio complicado» que uniría estos dos conceptos. De esta forma, la idea de nacionalismo es impensable sin la idea de democracia, y la democracia nunca existe sin nacionalismo. Apunta así a la vinculación directa del nacionalismo con el concepto capital de soberanía popular y como esta interconexión se materializó en un tipo de unidad política que pasaría a convertirse en molde imprescindible para la propagación de la idea democrática. Para Nodia, sólo el pueblo entendiéndose a sí mismo como «la nación» proporcionaba la cohesión política necesaria que era requerida por la democracia.

Otros autores se han apresurado en resaltar la idea de igualdad y cómo ésta, de forma implícita, viene aparejada al surgimiento de las ideas de nación y nacionalismo. A grandes rasgos, tras la fuerte irrupción del nacionalismo, la ciudadanía pasaba a entenderse ahora como un atributo del individuo, y dado que este atributo es el mismo para todos, la nacionalidad hace a todos los individuos potencialmente iguales. De esta forma, la emergente ciudadanía de carácter nacional ya no estaba confinada a los miembros de ciertas familias o personas de un alto estatus, sino que fue extendida también a los miembros de las clases bajas ${ }^{1}$.

Brubaker (1992, pp. 35-48) por su parte, se refiere a la Revolución francesa como un hito histórico que en síntesis inventó el Estado nación y la institución moderna de la ciudadanía nacional. La revolución presentaba una vertiente nacional que coexistió con otra vertiente democrática. El autor alude así a la importancia de la igualdad como una nueva realidad instaurada tras la revolución y su vinculación al carácter nacional de la misma: «As a national revolution, the French Revolution shaped the institution of modern citizenship in several distinct ways. By levelling legal distinctions inside the nation, it gave a common substance to citizenship: civil equality».

1 HELBLING (2009), p. 4. 


\section{NACIONALISMO SEGÚN LIAH GREENFELD}

Posiblemente la estudiosa del nacionalismo que más ha resaltado la estrecha relación existente entre nacionalismo y democracia sea Liah Greenfeld (1992, p. 10). Para la teórica, el ubicar la soberanía en el seno del pueblo, junto al reconocimiento de la igualdad fundamental entre los diferentes estratos, aparte de constituir el contenido de la esencia de la idea nacional moderna, se erige al mismo tiempo en principios básicos de la democracia.

En su desarrollo teórico, la idea de igualdad, implícita en la nueva realidad cultural surgida tras la expansión del nacionalismo, ocupa un papel fundamental. La autora señala fecha y lugar para el nacimiento del nacionalismo. Es en la Inglaterra del siglo XVI cuando, como resultado de una nueva entidad geopolítica (la nación), la existencia de una conciencia y realidad nacional eran ya un hecho.

Greenfeld define pues a la nación como una comunidad soberana e inclusiva con una membresía no afectada por las divisiones de clase y estatus, por tanto, igual y que es el objeto natural de la lealtad y compromiso de sus miembros. En este sentido y haciendo hincapié en la idea de igualdad que se desprende del concepto de nación, la autora subraya como cada miembro de la comunidad así interpretada, toma parte en su condición superior de élite y como consecuencia de ello, una población nacional estratificada es percibida como esencialmente homogénea y las líneas de clase y estatus se perciben como superficiales. Por ello, un fenómeno como el de la movilidad social, común en las democracias liberales actuales, constituye para Greenfeld un valor político directamente ligado y lógicamente derivado del nacionalismo.

Para la autora, la aparición de la nueva imagen nacional de la realidad (conciencia nacional o nacionalismo) inauguró una era de continuo cambio social orientado hacia la realización de los principios de la soberanía popular, la igualdad fundamental entre los miembros de la nación y de la identidad inclusiva implícitos en el nacionalismo. Estos principios, según Greenfeld, son mejor conocidos hoy en día bajo el nombre que les fue dado durante la Revolución francesa: libertad, igualdad y fraternidad ${ }^{2}$. El estallido de aquella revolución marcaba así la efectiva importación del nacionalismo a Francia y su propagación más allá de las islas británicas.

\section{EL AUTONOMISMO EN ARCHIPIÉLAGOS ATLÁNTICOS COMO UN FENÓMENO NACIONALISTA}

Dentro de esta vinculación entre nacionalismo y democracia, el artículo se interesará especialmente por un supuesto específico que apunta hacia una estrecha correspondencia entre el fenómeno nacionalista y una vocación autonomista para un tipo específico de archipiélago localizado en aguas del Atlántico, representado por Azores y Canarias. Consolidado hoy en día el autonomismo en ambos archipiélagos como una realidad refrendada por la gran mayoría de sus habitantes, su origen en las postrimerías del siglo XIX pocas veces ha sido analizado exclusivamente bajo la perspectiva del ideario nacionalista. Tal ideario resulta pertinente pues ya durante el siglo XIX se había convertido en una imparable fuente ideológica que impregnaba la esfera política y social de la Europa occidental.

Tomando en consideración la conexión entre nacionalismo y alguno de los principios básicos de la idea de democracia, se revisarán las manifestaciones autonomistas surgidas, casi al unísono, en dos archipiélagos con características geográficas, territoriales e histórico-políticas muy similares. Cada uno representa para el otro su realidad más parecida posible. Se procederá a esbozar la relación de los dos archipiélagos con el nacionalismo a lo largo del siglo XIX. Bajo este enfoque novedoso, se analiza la irrupción de demandas autonomistas en las Azores y Canarias a partir de la última década del siglo XIX. El presente trabajo propone que, ante todo, aquellas demandas han de ser concebidas como una derivada directa de la irrupción del ideario nacionalista, pese a estar guiadas por una serie de principios fácilmente identificables con la idea de democracia.

2 GREENFELD (2018), p. 148. 
El siglo XIX fue testigo del surgimiento de una identidad nacional de alcance popular para Portugal. El nacionalismo penetra en aquel territorio ibérico de la mano de España, que ya a comienzos del siglo XIX, en el contexto de la ocupación napoleónica, había experimentado su propia revolución nacional. Es en el año 1822, cuando el nuevo espíritu nacional portugués queda consagrado con la aprobación de la primera constitución democrática para Portugal ${ }^{3}$. No obstante, ya durante aquella década empieza a adivinarse una profunda brecha dentro del mapa político portugués, que acabará afectando a la cohesión interna de la recién concebida nación.

Las luchas por el poder entre los herederos al trono, desatadas durante el primer cuarto del siglo XIX, presentan una importante vertiente ideológica en cuanto que suponen también una agria confrontación entre los ideales liberales y los conservadores, siendo los miguelistas los patrocinadores de esta última corriente. Evidentemente aquel enfrentamiento ideológico también tenía un reflejo en la forma de concebir la nación de la que todos formaban parte. De esta forma la percepción interna de la nación quedaba fragmentada. Portugal se ve inmersa entonces en un período de gran inestabilidad con la aparición de sucesivos enfrentamientos armados, continuos cambios de gobierno y gran convulsión social.

En el último cuarto de siglo del XIX se llega a una nueva etapa de reconciliación en la que la inestabilidad, las agresiones y el enfrentamiento continuo pasan a ser hechos del pasado. Es en esta etapa cuando poco a poco se va entretejiendo una cohesión popular en torno a una identidad nacional cada vez con menos fisuras. Esta unificación del nacionalismo portugués tendrá lugar en torno a los valores del republicanismo que había comenzado a consolidarse con fuerza entre las clases intelectuales y populares desde las últimas décadas del siglo XIX. A su alrededor las dos corrientes anteriormente enfrentadas parecieron fusionarse a pesar de algunas escaramuzas por seguidores monárquicos que hicieron temer el volver a la confrontación. La crisis del ultimátum británico acaecida en el año 1890 constituyó un hecho traumático para los portugueses y pasó a erigirse como punto de inflexión en el que quedaría encarnada la unificación de la identidad nacional en torno a los principios del republicanismo, resultando ser una crisis que desgastó sobremanera la figura del monarca portugués $\mathrm{y}$, con ello, la propia institución de la monarquía ${ }^{4}$. A parte de la cohesión experimentada en torno a los valores republicanos, la identidad nacional portuguesa en aquel período se vio a su vez alimentada de una forma transversal, incluso en sectores contrapuestos, de una fuerte nostalgia imperial ${ }^{5}$ que evocaba un pasado glorioso de la nación portuguesa ligada a los descubrimientos, a su misión civilizadora y a su antaño dominio de los océanos como primera potencia marítima del mundo.

\section{PENETRACIÓN DE LA IDEA NACIONAL EN LAS AZORES}

Dentro de este contexto portugués del siglo XIX, las Azores pronto se significan como uno de los escenarios claves en los que se desarrollarán episodios trascendentales de los primeros años de vida de la recién inaugurada realidad nacional portuguesa. Los enfrentamientos fratricidas por arrebatar el poder del reino se reproducen en las islas y la confrontación ideológica conllevó que algunas de las islas se convirtieran en importantes enclaves liberales. En aquel momento las Azores adquieren una mayor relevancia como punto geoestratégico de suma importancia para el ente político portugués, más aún tras la independencia de Brasil ocurrida en 1822.

En las Azores se produce un exitoso e inmediato acomodamiento de la identidad nacional portuguesa, primero entre sus élites y oligarquías y posteriormente entre el resto de estratos. En esos primeros años de la experiencia nacional en Portugal, la isla de Terceira en 1832 llegó incluso a acoger la sede de la capitalidad del reino en la ciudad de Angra, eso sí, debido a unas circunstancias excepcionales. En todo caso, quizás el elemento más decisivo que propició la fácil acomodación de la identidad nacional portuguesa en las islas fue la propia historia del

3 Véase BIRMINGHAM (2005), p. 118; PEREIRA (2013), p. 49; CAMPOS (2016), p. 62.

4 CARVALHO (2012), p. 132; ESTEVE (2017), p. 283.

5 NEVES (2006), p. 2; POLANAH (2011), p. 42. 
archipiélago, que ya en el pasado - pero que también ahora en el presente y en un futuro no muy lejano - vinculaban estrechamente a las Azores, no sólo con Portugal como entidad política histórica, sino con lo que acabaría por convertirse a finales del siglo XIX en la esencia de la identidad nacional portuguesa. Elementos consustanciales a la historia y naturaleza del archipiélago como la heroica resistencia frente a los anhelos expansionistas de Castilla, escenificada en la severa derrota infringida a las tropas de Felipe II en la batalla de Salga (1581); el papel decisivo de las islas como escenario fundamental sobre el que se librarían los primeros conflictos que moldearían la historia nacional portuguesa y elevarían al archipiélago a la condición de bastión de la resistencia liberal; o la arrolladora naturaleza atlántica del archipiélago, que casaba con el linaje de potencia marítima que ansiaba restaurar la conciencia nacional portuguesa; todo ello, junto con el papel decisivo desempeñado por intelectuales y políticos azorianos como Antero de Quental, Teófilo Braga o Manuel de Arriaga en la consagración a nivel estatal de un republicanismo que acabaría por apuntalar al nacionalismo portugués a finales del siglo XIX, marcarían de una forma clara y concisa las inclinaciones nacionales dentro del archipiélago en favor de la identidad nacional portuguesa.

Prueba de esta filiación son las continuas manifestaciones de patriotismo hacia Portugal, expresadas públicamente por muchos de los representantes del primer movimiento autonomista azoriano. Tales manifestaciones no dejaban dudas del afianzamiento inequívoco de la identidad nacional portuguesa en territorio isleño.

De esta forma, durante la visita real efectuada en 1901 por el rey Don Carlos a las Azores en plena vorágine autonomista, destaca Cordeiro (2001, p. 10) cómo el contenido de los discursos oficiales deja claro que tal acontecimiento histórico se desarrolló en un clima de armonía, patriotismo y lealtad. Los responsables políticos y administrativos locales no cesaron de apelar a la historia, destacando el papel del pueblo azoriano en la defensa de la independencia nacional y los principios de la libertad ${ }^{6}$.

\section{SURGIMIENTO Y DESARROLLO DEL NACIONALISMO ESPAÑOL DURANTE EL SIGLO XIX}

Las guerras que tienen lugar en España a comienzos del siglo XIX con motivo de la ocupación napoleónica de la península ibérica desencadenarán importantes consecuencias sobre la población española en los órdenes político y social. Aparte de la irrupción con fuerza de los idearios liberales, la ocupación francesa y el subsiguiente levantamiento popular para recuperar la independencia del territorio, acabaron por implantar una perspectiva nacional sobre importantes sectores de la población española ${ }^{7}$. Al igual que en Portugal, para el caso español también pronto se distinguen dos bandos opuestos que amenazan con fracturar el mapa político español. Enfrentados igualmente en su origen por disputas sucesorias, aquel enfrentamiento tiene como materialización más reconocible una fuerte escisión ideológica entre conservadores y liberales que, a medida que vaya avanzando el siglo, se traduciría en una identidad nacional española profundamente dividida. Muro y Quiroga (2005, p. 15) se refieren a dos versiones del nacionalismo español presentes a lo largo del siglo XIX, una liberal y otra tradicionalista, que a su vez se encontrarían divididas internamente en diferentes grupos. Las dos corrientes en disputa, aunque coincidían en la afirmación de una identidad nacional común para todos los españoles, producían una honda fragmentación dentro de aquella nueva comunidad política definida ahora en términos nacionales. Paralelamente, las revoluciones nacionales acaecidas en las posesiones americanas durante el primer cuarto del siglo XIX, instauraron en la conciencia colectiva de los españoles ibéricos una sensación de constante cuestionamiento de su entidad nacional. Esta percepción de constante cuestionamiento se enquistaría con las primeras sublevaciones nacionales ocurridas en Cuba y Puerto Rico en la segunda mitad del siglo XIX, pasando a integrarse finalmente como uno de los rasgos definitorios de la identidad nacional española, superando la escisión interna, cuando aquel cuestionamiento llegó a la península ibérica en la forma de los nacionalismos catalán y vasco.

6 CORDEIRO (2001), p. 10

7 EASTWOOD (2006), p. 23; ÁLVAREZ JUNCO (2013), p. 308. 
El desastre del 98 aparte de constituir una crisis en la conciencia nacional de los españoles, también se significó como un punto de inflexión y motor que provoca una frágil unificación del nacionalismo español y de sus visiones enfrentadas, en torno a dos componentes de amplio consenso: el primero de ellos apuntaba al legado de potencia global hegemónica e influyente como un elemento consustancial a la naturaleza de la nación española, mientras que el segundo se relacionaba con una obsesión con la unidad física de la nación, reflejada en una defensa a ultranza de la integridad del territorio español que había sobrevivido al desastre del 98. Aparte de estos dos elementos, pocas cosas en común tenían las dos facciones de la identidad nacional española. No obstante, la progresiva consolidación de los nacionalismos periféricos junto al inminente riesgo de escisión percibido de una forma subjetiva por los nacionalistas españoles por estar imbuidos de la conciencia colectiva del eterno cuestionamiento, contribuyeron al enraizamiento definitivo de aquella unificación ficticia en torno a esos dos elementos, unificación que demostró una gran resiliencia durante todo el siglo XX.

\section{ENCUENTRO DE LA IDEA NACIONAL CON CANARIAS Y SU FRUSTRADA IMPLANTACIÓN POPULAR}

Canarias inicia el siglo XIX en medio de una gran convulsión, primero debido al estallido de la guerra de independencia en la península y las importantes transformaciones que trajo aquel conflicto a la sociedad española en general y, en segundo lugar, debido a la irrupción del pleito insular como un fenómeno que a partir de entonces se convertiría en la primera fuente de conflicto, impulso y activismo político entre las élites y oligarquías isleñas. En ese momento, las rivalidades internas quedan del todo patentes y se visualizarán en su forma más nítida en 1808 con la crisis de la Junta Suprema de Canarias, desencadenándose una encarnizada disputa entre la Junta Suprema constituida en Tenerife y el Cabildo Permanente de Gran Canaria. De esta forma, el proceso de construcción y articulación político-institucional de Canarias, que hasta entonces se había llevado a cabo a través del reparto que desde la España peninsular se hacía de las distintas cuotas de poder, ahora de una forma visible y descarnada, al quedar el conflicto insular al desnudo y habiendo alcanzado las distintas élites isleñas cierta madurez, no sólo quedará afianzado, sino que ahora los elementos insulares intentarán intervenir directamente durante todo el proceso de asignación de poder. La crisis de la Junta Suprema de Canarias marca la aparición de una actitud proactiva y deliberada desde sectores ya arraigados en las islas, los cuales operarían sin distracciones ni competencias ante una mayoría de la población desestructurada e inmersa en una realidad absorbente difícil de eludir. Así pues, el siglo XIX estuvo dominado en la esfera política de las islas por una agria confrontación entre los representantes de Tenerife y los de Gran Canaria, la cual se vio agravada en 1833 tras la designación de las islas como provincia única con capital en Santa Cruz de Tenerife. Como única excepción, cabe mencionar el hito que representó la declaración de puertos francos de los puertos canarios en 1852, quizás el único factor de cohesión identitaria entre las oligarquías y burguesías canarias, eso sí, en un plano puramente económico.

La sombra alargada del pleito insular como trasfondo que preside las relaciones políticas y de poder dentro de las islas durante todo el siglo XIX, supuso un obstáculo evidente para la penetración de la identidad nacional española de forma mayoritaria en la población canaria. Las élites y oligarquías, encargadas en principio de diseminar los postulados nacionalistas y de servir como referentes de una nueva cultura pública que emanaba de la ahora concepción nacional de España, se veían inmersas en una espiral de confrontación y rivalidad en ausencia de mediadores, llevando a cabo una movilización continua de los amplios recursos a su alcance (propaganda, prensa, iniciativas legislativas, acción política...) en favor de la causa insularista. Igualmente, la división interna de la identidad nacional también se significaría como una importante interferencia que dificultó el trasvase de la identidad nacional española desde las élites y oligarquías canarias hacia el resto de la población.

Aunque el pleito insular y la división interna del nacionalismo español se erigen como obstáculos más evidentes, no fueron los únicos presentes entre las élites y grupos influyentes de las islas durante las décadas de desarrollo y consolidación de la identidad nacional española a nivel popular. El interés que empieza a suscitar el mundo indígena dentro de la gente de la 
cultura durante el siglo XIX supone también un factor que distorsiona la acogida de la nueva identidad nacional. Aquí sobresale la denominada Escuela regionalista de La Laguna con autores como Zerolo, Tabares Bartlett, Martínez de Escobar o Perera. Surgida en el último cuarto del siglo XIX, presenta como uno de sus hilos conductores la idealización del mundo indígena. Por su parte, el campo de las ciencias también despierta el interés sobre los antiguos habitantes de las islas, como destacan los estudios de figuras como Berthelot, Bethencourt Alfonso, Chil Naranjo o Millares Torres. Es importante remarcar como alguno de estos autores comienzan a señalar en sus obras la continuidad biológica y cultural indígena en la población y sociedad isleña posterior, consolidándose así, bajo el amparo de la ciencia, una identidad cultural genuina de las islas. En medio de un clima de reivindicación de la cultura guanche, de exaltación de sus valores, así como de una gran producción de trabajos de investigación que tratan el pasado indígena ${ }^{8}$, el talante imperial y civilizador preconizado por la unificación del nacionalismo que venía de la península situaba a los canarios más como un producto de aquella historia gloriosa y vigor hegemónico que como parte de aquel, esto es, más como objeto que como sujetos.

También los primeros años del siglo XX son testigos del surgimiento de un regionalismo canario que tiene como una de sus grandes expresiones escritas la obra Regionalismo en las Islas Canarias. Estudio histórico, jurídico y psicológico publicada en 1904 por Manuel de Ossuna. Aquel empuje regionalista tuvo su primera materialización en la creación en 1908 de la Liga Regionalista en el Puerto de la Cruz, la cual llega a mantener contactos con Cambó y la Lliga ${ }^{9}$.

Finalmente debe mencionarse, aunque con un mínimo impacto en suelo isleño, el surgimiento de un nacionalismo canario entre la colonia de emigrantes isleños establecida en Venezuela. Esta corriente encontró su vía de expresión en la revista quincenal El Guanche que comenzó a publicarse en noviembre de 1897 en tierras venezolanas. Al frente de esta publicación se encontraba la figura de Secundino Delgado y los palmeros Guerra Zerpa y Brito Lorenzo. Esta primera manifestación de un nacionalismo canario englobaba a sectores de la pequeña burguesía local emigrada a América junto con miembros de los núcleos obreros revolucionarios ${ }^{10}$.

Mas allá de la rivalidad política entre islas, en el último cuarto del siglo XIX, la sociedad canaria se mostraba ampliamente fragmentada en cuanto a qué comunidad específica de individuos (estado, región o isla) y en qué grado debía ser objeto de la lealtad colectiva y solidaridad política. Existe una atomización de lealtades, intereses e identidades entre las oligarquías, élites y grupos de influencia del archipiélago. Las élites capitalinas, principales protagonistas del pleito insular, tejían unas redes de lealtad y compromiso a nivel insular. A pesar de ser un grupo «españolista» ${ }^{11}$ movilizaban todos sus recursos con el fin de sobresalir regionalmente o contrarrestar el poder de la otra isla. La, hasta finales del siglo XIX, divida identidad nacional española habitaba en ellos en una esfera meramente privada sin transmisión generalizada al ámbito público.

Por su parte, sectores destacados del mundo intelectual y cultural desarrollaban unas aspiraciones cada vez más regionalistas a la par que exploraban una identidad cultural propia de las islas. Dentro de aquel contexto de introspección, de forma inevitable, surgía el mundo indígena y su legado como fuente originaria del nexo de singularidad que unía a los habitantes del archipiélago. La revisión de la historia indígena desde las islas evidenciaba la especificidad de la realidad canaria y su inconexión frente alguno de los postulados que acabarían erigiéndose como núcleo básico de la identidad nacional española tras el desastre del 98.

La identidad nacional española, por tanto, a finales del siglo XIX, quedaba relegada en Canarias a una minoría representada por miembros de las grandes élites capitalinas y de la clase media isleña. Ello fue como consecuencia de la profunda escisión interna que arrastró el nacionalismo español durante todo el siglo XIX, de la lucha encarnizada que enfrentaba a las élites y oligarquías isleñas en el contexto del pleito insular y de la particularidad geográfica,

8 GARÍ (1992), pp. 57-59.

9 HERNÁNDEZ BRAVO DE LAGUNA (2004), p. 14.

10 HERNÁNDEZ GONZÁLEZ (1998), p. 6.

11 GONZÁLEZ (1998), p. 12. 
histórica y étnica del archipiélago propensa al surgimiento de identidades colectivas de carácter político o cultural que rivalizaban con la identidad nacional española entre las clases medias, intelectuales y colonias de emigrantes en América. Ello se complementa con otros factores más estructurales, como la debilidad de las instituciones estatales destinadas a nacionalizar el país (escuela, servicio militar universal, industrialización...). Todo esto se traducía en una indefinición, si no endeblez, de la comunidad nacional, en una incongruencia entre la unidad política y la unidad cultural, así como en una manifiesta imposibilidad de que pudiera consagrarse entre las élites y principales estratos del archipiélago una solidaridad política y lealtad colectiva suprema, como así exigía el nuevo ideario nacionalista.

El proceso mental por el cual un individuo pasaría a concebirse como miembro de una nación, con todo lo que ello comportaba, no se da entre la población canaria de una forma generalizada, ni a través de una identidad nacional española que contaba con el respaldo del aparato estatal, ni a través de una identidad nacional específica para las islas. Mas allá de aquella importación abortada, los principios inherentes a la idea de nacionalismo compartidos por el concepto de democracia, serán percibidos de una forma abstracta e indeterminada por aquellos que habían asumido cualquiera de las identidades nacionales disponibles en el archipiélago. En ningún caso tuvo lugar una percepción generalizada del conjunto de las islas como sujeto específico depositario de aquellos principios e ideas, ya fuera por considerarse una mayoría de la población isleña como parte integrante de la nación española o por concebir una mayoría de isleños al archipiélago como una realidad nacional propia.

Ante este vacío de referentes que pudieran activar la lealtad colectiva y solidaridad política del isleño, el pleito insular y las dinámicas asociadas a éste crecieron con fuerza. El pleito se hizo grande, entre otras cosas, debido a la ausencia en el seno de la población canaria de un factor cohesivo y transversal de orden político que pudiera haber actuado como contrapeso, factor que, según la referencia a Nodia del primer epígrafe, quizás pudiera haber estado representado por el nacionalismo.

\section{EL PRIMER MOVIMIENTO AUTONOMISTA AZORIANO}

Es a finales del siglo XIX cuando acontece el primer movimiento en pro de una autonomía para las Azores. La última década del siglo XIX tuvo unas consecuencias políticas de gran calado para la realidad de las islas y, sobre todo, para la apreciación que los isleños comenzaban a tener del territorio que habitaban. El primer movimiento autonomista acabaría convirtiéndose en el germen de una futura percepción colectiva que llevaría a concebir al archipiélago en su conjunto como un sujeto político determinado.

Un importante factor, considerado como imprescindible para la aparición de aquel movimiento autonomista, sería la propia adopción de la identidad nacional portuguesa por una mayoría de la población azoriana. Una de las consecuencias más decisivas de aquella conversión del pueblo azoriano a la causa nacional fue la asimilación y afianzamiento entre la sociedad isleña de los principios políticos dimanantes de la ideología nacionalista, esto es, la soberanía popular, la igualdad fundamental entre los miembros de la nación y la identidad inclusiva.

El enraizamiento en el archipiélago de una identidad nacional y, con ella, la interiorización y asimilación de los principios que comportaba, conllevarán que muchos azorianos influyentes empiecen a mirar con ojos críticos su relación de soberanía con Lisboa con base en la repetida percepción y constatación de un trato desigual entre los portugueses continentales y los portugueses insulares. Así, Amaral (2011, p. 214) apunta el hecho de que una de las principales líneas de pensamiento dentro de la tradición azoriana relaciona de una forma directa el desarrollo de una identidad insular con el propio estándar de vida de los isleños, cuando este era comparado con el estándar de vida de los portugueses continentales. Según Amaral, una noción identitaria surgiría en las Azores durante la segunda mitad del siglo XIX, que proveería a los azorianos con una conciencia activa de las dificultades y dureza de la vida isleña, particularmente cuando ésta era comparada con la vida de aquellos que residían en la Portugal continental. Para el autor, se trataba de una identidad anclada en un profundo resentimiento de 
los isleños vis-à-vis sus compatriotas continentales. Durante la segunda mitad del siglo XIX, las voces críticas de la población azoriana crecieron en número, afirmando que la estrategia nacional seguida por el Estado no sólo era incapaz de responder a las necesidades específicas del archipiélago, sino que iban en contra de los propios intereses isleños ${ }^{12}$.

Ligado a su vez con el período de regeneración que vivió Portugal durante la segunda mitad del siglo XIX, los instrumentos ideológicos con los que el nacionalismo había equipado a los azorianos transformaron aquella etapa histórica en las islas en un periodo de reivindicación y conciencia popular. Es, precisamente, la ausencia de efectos de la regeneración en tierras azorianas lo que, en última instancia, para algunos autores, contribuye de una forma decisiva a la aparición del primer movimiento autonomista en las islas ${ }^{13}$. Cordeiro se muestra bastante tajante en esta aseveración y sostiene que factores como la propuesta de establecimiento de un monopolio del alcohol en favor del gobierno o el intento de supresión del curso diferenciado de la moneda en las islas u otras medidas, que suponían un agravamiento de las cargas fiscales de los azorianos, representaban tan sólo factores menores a los que el autor denomina de orden coyuntural y que, según aquél, por sí mismos, no bastaban para explicar la magnitud del proceso reivindicativo azoriano de la década de 1890. Para Cordeiro (1992, p. 265), las causas más profundas que explicaban aquel proceso debían de buscarse en el advenimiento de la regeneración. De esta forma, amplias capas de la población isleña empezaron a advertir una desigualdad de trato, al considerar que el esfuerzo desarrollista entrante en el continente era verdaderamente insignificante para el caso de las Azores, al mismo tiempo que se comenzaba a tener una percepción entre las élites azorianas de que se trataba de limitar alguna de las ventajas con las que contaban las islas.

De entre las figuras más importantes que protagonizaron el primer movimiento autonomista, destaca la del abogado y político Aristides Moreira da Motta (1855-1942), considerado el padre de la autonomía azoriana. El punto culminante en su carrera política lo constituyó la presentación de una propuesta de autonomía para las Azores ante la Asamblea Nacional Portuguesa en 1892. Designó a la propuesta como La libre administración de las Azores por los azorianos, título que acabaría por convertirse en el lema del primer movimiento autonomista. Moreira da Motta fundamentaba su activismo en pro de una autonomía para las Azores en la situación singular de sus habitantes, los cuales vivían en una realidad geográfica única que había conducido a un ambiente social único. En líneas generales, el líder autonomista defendía que los azorianos poseían una forma de ser, individual y colectivamente, que era diferente de la experimentada por la población portuguesa en el continente ${ }^{14}$.

Moreira da Motta sitúa como corolario de la instauración de un régimen autonómico la conquista de la igualdad de los ciudadanos azorianos en relación a los portugueses del continente. Para este, sólo la autonomía que posibilita la adaptación de las leyes a las realidades particulares de la vida insular permitiría la conquista de la igualdad de los ciudadanos azorianos con respecto a sus compatriotas continentales, sobre todo en términos de calidad de vida ${ }^{15}$.

El movimiento acabaría desencadenando la aprobación en Lisboa del Decreto de 2 de marzo de 1895 por el que se dotaba a los distritos isleños de mayores cuotas de autonomía para su organización, aunque el camino hasta este logro no fue fácil.

Sin duda, el principal obstáculo con el que se topó el primer movimiento autonomista fue la división interna del archipiélago debido a la organización político administrativa de las Azores, fundamentada históricamente sobre la entidad política isla. Además, la historia isleña estaba salpicada de desavenencias y desconfianza entre las islas más importantes: São Miguel, Terceira y Faial.

De esta forma, el primer movimiento autonomista se encuentra muy centrado en la isla de São Miguel y tiene un menor peso en el resto de islas con la excepción de Terceira ${ }^{16}$. Los autonomistas de São Miguel, a pesar de que intentaban extender sus reivindicaciones a las islas de Terceira y Faial, debían hacer frente a las reservas o indiferencia de los círculos políticos e

12 COSTA (2008), p. 249.

13 CORDEIRO (1992), pp. 263-265; COSTA (2008), p. 249.

14 COSTA (2008), p. 250.

15 CORDEIRO (1992), p. 277.

16 COSTA (2008), p. 250. 
intelectuales de aquellas dos islas. Para Abrunhosa (2008, p.146), el primer movimiento autonomista azoriano se vio limitado por una incapacidad de llegar por igual a todas las islas.

Igualmente es importante reseñar como, en general, la perspectiva autonomista era una perspectiva distrital, por tanto, de corto alcance, sin una proyección sobre el conjunto del territorio azoriano e íntimamente relacionada con el empoderamiento de determinados centros urbanos. La conciencia autonomista era distrital y no azoriana ${ }^{17}$.

A pesar de ello, el primer movimiento autonomista fue testigo de esfuerzos entre las islas por conseguir lo que se entendía como un objetivo político compartido y, de hecho, se llevaron a cabo visitas de delegaciones de unas islas a otras para intentar alcanzar un consenso con el fin de alzar una voz común en favor de la autonomía azoriana. Igualmente, el primer movimiento autonomista llevó al pueblo azoriano y, en concreto, a sus élites políticas e intelectuales a ser conscientes de la división interna que existía entre las islas y la necesidad de fraguar una identidad común entre ellas. Esta necesidad se hará patente e intentará abordarse durante el segundo movimiento autonomista azoriano ocurrido en la segunda década del siglo XX.

\section{VOCES AISLADAS EN FAVOR DE UNA AUTONOMÍA CANARIA A COMIENZOS DEL SIGLO XX}

En Canarias no tuvo lugar una reivindicación autonomista de las dimensiones de la experimentada en las Azores durante la última década del siglo XIX. No obstante, en los años inmediatamente posteriores al movimiento azoriano, empiezan a alzarse voces aisladas en Canarias en favor de una autonomía política para el archipiélago. Son expresiones que saldrán a la luz pública principalmente a través de publicaciones, ya fueran diarios o revistas, y aunque no tuvieron un impacto decisivo en la población isleña, sus emisores deben considerarse como los precursores de una concepción de las islas en la que éstas aparecían unidas políticamente bajo el paraguas de la autonomía, concepción que con el paso del tiempo llegaría a establecerse y arraigarse con fuerza en la sociedad canaria. A continuación, se tratará con más detalle algunas de las figuras más representativas que reivindicaron una autonomía para el archipiélago en los inicios del siglo XX.

\section{Secundino Delgado (1867-1912)}

De entre aquellas figuras más significativas, debe mencionarse a Secundino Delgado. Importante figura de la historia política canaria, más por su legado que por la trascendencia que pudo tener en vida, el autodidacta Secundino fue manifiestamente influenciado por la Escuela regionalista de La Laguna ${ }^{18}$. En numerosas ocasiones designado como líder fundador del nacionalismo canario, pocas veces se ha resaltado su faceta como autonomista.

Secundino comienza una activa campaña autonomista cuando regresa a las islas en 1900 después de su periplo por América. Su predicar autonomista se desarrolla desde una doble vía, por una parte, a través de los artículos publicados en la revista Vacaguaré fundada en 1902 y, por otra, mediante la política de partido encarnada en su militancia en el Partido Popular Autonomista. El autonomismo de Secundino emana esencialmente del hecho de concebir a las islas como una nación que en su día fue sometida por la fuerza y privada de libertad. De este pensamiento se derivaría de forma natural el derecho de autogobierno de las islas; todo ello también de acuerdo con la naturaleza de los tiempos que imponían como signo de progreso los principios emanados de la Revolución Francesa como la igualdad y la justicia ${ }^{19}$. De esta forma, Secundino no cesa de referirse al ejemplo de otras naciones cultas como Inglaterra que se habían adelantado en otorgar a sus colonias y provincias amplia autonomía. Esta idea de autogobierno, referida a las Canarias gobernadas por y para los canarios, conduciría entonces a la ansiada liberación del archipiélago que como antaño volvería a ser libre.

17 ABRUNHOSA (2008), p. 149.

18 HERNÁNDEZ BRAVO DE LAGUNA (2004), p. 13.

19 HERNÁNDEZ GONZÁLEZ (2006), p. 493. 
En el pensamiento de Secundino también aparecen ideologías radicales de izquierda como el anarquismo pero, a pesar de ello, en sus numerosos escritos para las diversas publicaciones en que intervino, se adivina antes que nada una completa anexión al ideario nacionalista. Precisamente, el personaje se presenta imbuido de aquellos preceptos que, según Greenfeld, se muestran inseparables de la idea nacional como la libertad, la igualdad y la voluntad/soberanía popular. Llevado a la práctica, Secundino hace un llamamiento a la movilización popular como la única vía para conseguir la emancipación y mejoramiento de la patria canaria.

Es de lógica y coherencia la demanda autonomista por parte de una figura que piensa en canario y que se distinguió durante toda su vida como un importante activista de la causa nacional isleña. Sin embargo, también surgieron importantes personalidades públicas, alineadas dentro de la identidad nacional española, que se convirtieron en vehementes defensores de una autonomía para las islas, como fueron los casos que aquí se expondrán de los palmeros Pedro Pérez Díaz y Luis Felipe Gómez Wangüemert y del granadino Ricardo Ruiz Aguilar.

\section{Pedro Pérez Díaz (1865-1930)}

Pérez Díaz fue jurista y figura de gran relevancia intelectual que puede ser enclavado dentro del establishment de la España de la Restauración. En este sentido, durante casi cuarenta años fue letrado del Consejo de Estado.

Los escritos del jurista palmero cuyo contenido incluían la opción autonomista en favor de las islas se sucedieron desde 1906 hasta 1911 en periódicos como el tinerfeño El Progreso o el palmero Germinal. Sin embargo, su obra en última instancia se significó, sobre todo, por su defensa de una autonomía meramente insular y no por sus alegatos en favor de una autonomía regional para Canarias, considerándose hoy en día su trabajo ensayístico como uno de los elementos que mayor inspiración ejercieron en la elaboración de la Ley de Cabildos Insulares aprobada en 1912. Todo ello a pesar de que Pérez Díaz, en un ensayo de 1906, defendiera ya «que por todos los medios debe procurar obtenerse la más amplia autonomía políticoadministrativa para esa provincia» ${ }^{20}$ (refiriéndose a Canarias) y que en 1912, a colación de la aprobación del Reglamento provisional sobre los cabildos insulares, escribiera que «el reglamento consagraba el régimen demandado por las islas, pero no era más que un punto de partida, puesto que el fin último era, en realidad, la autonomía regional» ${ }^{21}$.

En los escritos de Pérez Díaz se observan continuamente los designios vinculados a una concepción del nacionalismo en que sobresalen principios muy cercanos al ideal democrático, de lo que se desprende que nos encontramos ante un autor con una firme perspectiva nacionalista. Como miembro de una nueva generación de krausistas y de republicanos centristas, se mostraba claramente contrario a los nacionalismos periféricos y en favor de la unidad de la nación española, pero dentro de un marco de respeto a la diversidad cultural e institucional que mostraba España ${ }^{22}$. Para Pérez Díaz, uno de los grandes problemas que asolaban el país durante aquella época era el elemento nacionalista del Estado español. Expresaba una necesidad por perfeccionar la nacionalidad española pues entendía que ésta se encontraba alejada de la vida popular, y era cada vez más extraña al pueblo. En este punto, según el autor, debería entrar en juego el Estado, que tendría como importante misión corregir aquella tendencia. La forma propuesta sería mediante la creación de organizaciones regionales dotadas de autonomía ${ }^{23}$.

De entre los ensayos de Pérez Díaz sobresale con fuerza el principio de libertad, entendido como un derecho natural que asiste a toda personalidad, ya fuera individual o colectiva, para que pueda realizar su vida. En algunas líneas incluso alude literalmente al concepto anglosajón de self government. Pero de entre todos los principios normalmente relacionados con el nacionalismo y compartidos por la idea de democracia, el principio de igualdad es el que tiene

20 PÉREZ DÍAZ (2017), p 39.

21 PÉREZ DÍAZ (2017), p. 24.

22 PÉREZ DÍAZ (2017), p. 16.

23 PÉREZ DÍAZ (2017), p. 57. 
un mayor protagonismo en sus escritos y el que parece elevarse como justificación última en favor de una autonomía regional. Remarca en numerosas ocasiones el gran error de trasponer la legislación que rige en la península a la estructura particular de las islas y señala que es ahí donde radica el conflicto y el origen de los problemas. El autor alude especialmente a la ley provincial como especialmente gravosa para los intereses de Canarias y lo inadecuada que es esta ley para las islas, ya que fue pensada y dictada teniendo en cuenta la realidad territorial peninsular. Para Pérez Díaz la organización que debe regir sobre las islas, debía ser ideada teniendo en cuenta específicamente tres elementos fundamentales de Canarias: su propia personalidad como islas, la vida de relación de las islas entre sí y la del archipiélago con el Estado $^{24}$.

\section{Ricardo Ruiz Aguilar (1839-1922)}

Ruiz Aguilar también podría ser encuadrado como miembro de aquel establishment al frente del periodo de la Restauración española. Nacido en Granada, la figura de Ruiz Aguilar presenta una dilatada carrera como militar. Un destierro por causas políticas le llevó a Tenerife en la segunda mitad del siglo XIX donde se emparentó con una familia de la nobleza isleña al contraer matrimonio con la hermana del Marqués de La Florida. También desempeñó una importante faceta como político como prueba su designación en 1896 como diputado a Cortes por la isla de Tenerife o el cargo de Gobernador Civil de Baleares que desempeñaría durante 1906.

La vertiente de este personaje que más interesa al presente artículo es la de difusor de la causa autonomista canaria. El militar granadino comienza una campaña a favor de la autonomía de las islas en el año 1901 desde las páginas del periódico de su hijo Las Canarias, publicado en Madrid. El autor inicia su primer artículo en pro de una autonomía canaria, estableciendo una definición clara de lo que él entiende por autonomía, y lo hace vinculando de una forma directa aquel concepto con la idea de libertad: «entiéndase por autonomía la condición en la cual un Estado o un individuo conserva, con entera libertad e independencia, aquello que constituye su manera de ser esencial, característica y propia» ${ }^{25}$.

Ruiz Aguilar explica que se apresura en dar una definición, ya que es consciente que la palabra autonomía «asusta». Pero el militar granadino considera que la concesión de autonomía, más que suponer un riesgo para la unidad territorial de la nación española, supondría un remedio en favor de aquella, que si se hubiese aplicado para el caso de Cuba habría evitado los dramáticos sucesos del 98.

Dentro del discurso autonomista de Ruiz Aguilar subyace también con fuerza una demanda de igualdad de trato para con las islas. Así, dedica la totalidad de un artículo a denunciar lo inadecuado que resultaba el hecho de que una legislación pensada y construida desde la España peninsular se aplicara a un territorio con una realidad tan específica como el canario, enumerando previamente a grandes rasgos las peculiaridades de cada una de las islas y de la política canaria en general. El autor entiende que el archipiélago canario no debía ser gobernado como una provincia más de la España continental y aboga por la instauración en las islas de procedimientos políticos y administrativos distintos.

Por último, habría que destacar también como el propio Ruiz Aguilar finaliza unos de sus escritos autonomistas haciendo una mención expresa de las islas Azores, «cuya legislación comencé a estudiar cuando fui diputado por Tenerife» ${ }^{26}$ advirtiendo como conclusión del mismo, que tal vez hubiera algo bueno que imitar de las posesiones portuguesas.

\footnotetext{
24 PÉREZ DÍAZ (2017), p. 98.

25 HERNÁDEZ GONZÁLEZ (2006), p. 470

26 HERNÁNDEZ GONZÁLEZ (2006), p. 472
} 


\section{Luis Felipe López Wangüemert (1862-1942)}

López Wangüemert fue un político, intelectual y periodista palmero que también se pronunció abiertamente por una autonomía para Canarias en los primeros años del siglo XX. Su vida transcurrió entre la isla canaria de La Palma y Cuba y su caso representa un fiel reflejo de la especificidad del fenómeno nacionalista en Canarias. De enormes convicciones patrióticas, participó en el bando español en la guerra de independencia de Cuba y organizó un escuadrón de voluntarios en favor de la causa española. Sin embargo con el paso del tiempo y debido en parte a las profundas decepciones que le produjeron sus anhelos políticos, nuestro personaje sufrirá una conversión que le acabará situando dentro de las filas del nacionalismo canario, hasta el punto de ser uno de los fundadores en La Habana del Partido Nacionalista Canario en 1924 y director de su órgano de prensa, la revista El Guanche (segunda etapa).

Mucho antes de aquella efeméride, López Wangüemert ya había publicado un artículo en septiembre de 1901 en el Diario de Avisos, en el que saludaba con satisfacción la creación en Tenerife del Partido Popular Autonomista. El intelectual palmero veía con buenos ojos que figurara en su programa la autonomía para el archipiélago, y señaló que Canarias, por «su posición geográfica y por otras causas que no son tratadas en esta sección, debe ser autónoma, quizás con más derecho que la industriosa y enérgica Cataluña ${ }^{27}$ ».

Justo un mes antes, en otro artículo publicado en el mismo diario, López Wangüemert denunciaba la situación de dejadez de la que estaban siendo objeto los habitantes de las islas de Lanzarote y Fuerteventura. En el texto se cita como, mientras el hambre más espantosa impera en Fuerteventura y Lanzarote, el rey Alfonso asistía a los toros y regalaba a los toreros sendas petacas de oro a la vez que el pueblo madrileño gritaba entusiasmado «iViva el Rey!». En este breve artículo se observan dos secuencias que dejan entrever interesantes matices de un pensamiento nacionalista perfectamente interiorizado por el autor. Por una parte, destaca la preocupación del palmero por la problemática de los habitantes de Lanzarote y Fuerteventura, lo cual apunta a la solidaridad archipelágica de la que tanto haría proselitismo durante toda su vida y que, sin duda, se constituiría en base para su futura conversión nacionalista. Por otro lado, subyace también con fuerza la denuncia al quebranto del principio de igualdad en relación con los españoles de las Canarias. En última instancia, a través de una pequeña anécdota se ponía de relieve la enorme desigualdad en las condiciones de vida entre miembros de una misma comunidad nacional: «los ministros y el pueblo se divertían en la plaza de toros ignorantes $\mathrm{u}$ olvidados de que perecían de inanición millares de sus súbditos, de sus gobernados, de sus hermanos en la nacionalidad» ${ }^{28}$.

López Wangüemert remarca la necesidad de una autonomía para Canarias como una vía efectiva y de derecho para superar el mal gobierno aplicado en el archipiélago desde la península. Como en el resto de autores identificados con la identidad nacional española, nuevamente se alegaba el principio de libertad como medio para la consecución de la igualdad con respecto al resto de territorios nacionales ${ }^{29}$.

Al igual que Secundino Delgado y Ruiz Aguilar, el palmero reclamaba la implantación de la autonomía por la propia naturaleza de los tiempos que así lo exigían e igualmente era de la opinión que su aplicación a tiempo en Cuba hubiese impedido el desastre posteriormente vivido en la isla antillana. Finalmente, del discurso autonomista de López Wangüemert se desprende también la necesidad de aplicar un sistema político que se adaptara a la realidad canaria, tal y como él lo explica «imponiéndole fisonomía propia, isleña» ${ }^{30}$. Se repite pues la idea en este autor en relación con el respeto de la diversidad y, en este caso, especificidad canaria, como requisito para la consecución de una igualdad real entre todos los miembros de la nación.

En todos los autores tratados aparecen de forma directa o indirecta aquellos principios ligados al fenómeno nacional que, a su vez, son compartidos por la idea de democracia según los teóricos del nacionalismo citados, si bien habría que destacar que para el caso del

27 PAZ SÁNCHEZ (1991), p. 104.

28 PAZ SÁNCHEZ (1991), p. 188.

29 PAZ SÁNCHEZ (1991), pp. 120, 192.

30 PAZ SÁNCHEZ (1991), pp. 120, 192. 
nacionalista canario Secundino Delgado, sobresale sobre el resto el principio de libertad, mientras que para el caso de los autonomistas con una identidad nacional española se esgrime con mayor intensidad la idea de igualdad. Ello obedece a que Secundino Delgado concibe el archipiélago como una nación sometida y diagnostica el encaje de las islas dentro del Estado español como el principal obstáculo que impedía el desarrollo y progreso del pueblo canario. Por su parte, los nacionalistas españoles hacen hincapié en la situación de desigualdad de la población isleña vis a vis con sus connacionales peninsulares, desigualdad que tenía su raíz en la aplicación sin filtro en el archipiélago de políticas diseñadas en función de la realidad continental. Por lo tanto, al no tenerse en cuenta las especificidades isleñas, aquellos autores identificaban una profunda desigualdad originaria en todo planteamiento político e institucional aplicable a la comunidad nacional en general. Tal planteamiento sí tenía en cuenta y se preocupaba por la realidad que afectaba a sus miembros en la península, pero no hacía lo mismo con la realidad singular a la que debían hacer frente sus miembros en las islas atlánticas. Se señalaba como resultado de la aplicación de esas políticas, una desigualdad entre los habitantes continentales y los isleños en perjuicio de éstos últimos, que quedaba reflejada en términos de calidad de vida o de eficacia de las políticas proyectadas. Se trataba pues de la denuncia de una doble desigualdad: una primera desigualdad en el punto de partida y una segunda, consecuencia de la primera, que era la que se hacía más evidente al materializarse en una gran diferencia en las condiciones de vida entre miembros de una misma comunidad nacional.

Detrás de gran parte de la reivindicación autonomista de Pérez Díaz, Ruiz Aguilar, López Wangüemert y Moreira da Motta, para el caso azoriano, es fácil adivinar un hilo conductor entre los respectivos planteamientos que apela a una lógica y razonamiento previsibles. Ambos son consecuencia de la total asimilación entre estas figuras del ideario nacionalista y, con éste, de una nueva forma de percibir la comunidad política de la que formaban parte, a través de la cual la igualdad fundamental entre sus miembros pasaba a convertirse en una de sus principales características definitorias.

\section{CONCLUSIONES}

Dentro de la esfera académica dedicada al estudio del nacionalismo son varios los autores que apuntan a la existencia de una estrecha relación entre el fenómeno nacionalista y la noción de democracia. Entre ellos, Greenfeld alude directamente a una coincidencia de principios entre aquellos que conforman el contenido de la esencia de la idea nacional moderna y aquellos que apuntalan el concepto de democracia. Se entiende que el movimiento autonomista de las Azores corrobora aquella relación. De esta forma, será la adopción de una identidad nacional (en este caso, la portuguesa) por una mayoría de la población isleña y, con ella, la interiorización de unos principios íntimamente ligados también con la idea de democracia como los de igualdad, libertad o soberanía popular, los que desencadenaron un movimiento a escala popular en aquel archipiélago durante la última década del siglo XIX. Paralelamente, es ilustrativo también, el sustrato común de las voces marginales que se mostraron activas en favor de una autonomía para Canarias en los primeros años del siglo XX. Estas se correspondían con figuras públicas ligadas de forma consciente o inconsciente con el dogma nacionalista, con lo que es fácil observar en sus escritos el reflejo de aquel dogma en la forma de un compromiso y exaltación continua de los principios compartidos por nacionalismo y democracia según el contenido de este artículo.

Este autonomismo residual, ligado directamente al fenómeno nacionalista, puede entenderse pues como una manifestación más del difícil tránsito de la idea nacional por Canarias. La forma que adoptó el autonomismo en Canarias en los albores del siglo XX coincide pues con la debilidad, variabilidad, mutabilidad y corto alcance de las ideas de nación y nacionalismo en el seno de la población canaria de entonces. Sin embargo, conviene remarcar la importancia del hecho de que aquellos autonomistas tratados se constituyen en punta de lanza dentro de la historia política de Canarias. Ello fue posible, como se desprende de sus escritos, a través de la consagración por todos ellos, como principal núcleo inspirador de las demandas autonomistas, de una serie de principios que, si bien coincidían con aquellos tradicionalmente relacionados 
con el concepto de democracia, habían sido interiorizados por estas figuras a través de una vía representada por la fuerte irrupción del nacionalismo en la península ibérica a comienzos del siglo XIX: encarnada esta vía en el compromiso y adscripción personal de cada uno de estos personajes a una determinada comunidad concebida como nacional, ya fuera la española o la canaria.

En ambos archipiélagos, el nacionalismo y los principios ligados a aquel, actuaron como fuerza inspiradora que preconizaba el cambio hacia una nueva forma de administrar políticamente cada uno de los territorios insulares. Sólo en las Azores adquiere la dimensión de movimiento debido a la canalización con éxito de la nueva forma de conciencia colectiva representada por el nacionalismo, más las ideas y principios ligados a aquel, en una única identidad nacional (la portuguesa) abrazada mayoritariamente por los azorianos.

El nacionalismo se presenta pues, en el caso de las Azores, como un motor de cambios alimentado por principios como la igualdad, la soberanía popular o la libertad, con un impacto sobre la práctica totalidad de la población, debido a su transversalidad e inclusividad.

Para el caso de Canarias, la mayoría de sus habitantes durante el período tratado no se percibían como miembros de una nación, ya fuera española o canaria. Por ello, no podía surgir en las islas una conciencia nacional colectiva o producirse una adhesión mayoritaria de la población canaria a la conciencia nacional española. Acontece en las islas una incapacidad de experimentar de forma grupal aquellos principios íntimamente ligados a las ideas de nación y nacionalismo compartidos a su vez por el concepto de democracia. Bajo estas circunstancias, parece especialmente complicado el que pudiera desencadenarse por iniciativa y determinación propias una acción colectiva inspirada por aquellos principios. Más aún, se dibujaba un escenario extremadamente propicio para que el pleito insular y la cultura política ligada a aquel, siguieran enraizándose con fuerza en la sociedad canaria.

\section{REFERENCIAS}

ABRUNHOSA, A. (2008). Precedentes histórico-teóricos dos regionalismos dos Açores e da Galizia. Tesis Doctoral de la Universidad de Santiago de Compostela. Recuperado el 01/10/2019 de

https://minerva.usc.es/xmlui/bitstream/handle/10347/2399/9788471914651_content.pdf?se quence $=1 \&$ isAllowed $=\mathrm{y}$

ÁLVAREZ JUNCO, J. (2013). «Spanish National Identity in the Age of Nationalisms». En M. A. CENTENO y A. E. FERRARO (Eds.), State and Nation making in Latin America and Spain. Nueva York: Cambridge University Press, pp. 307-328.

AMARAL, C. E. P. (2011). «Decentralization and Asymmetries in Portugal». En F. REQUEJO y K. NAGEL (Eds.), Federalism beyond Federations. Surrey: Ashgate Publishing Limited, pp. 203-222.

BIRMINGHAM, D. (2005). Historia de Portugal. Madrid: Ediciones Akal, S.A.

BRUBAKER, R. (1992). Citizenship and Nationhood in France and Germany. Cambridge, USA: Harvard University Press.

CAMPOS, S. (2016). «Tradition and Modernity in Portuguese Liberal Political Culture on the Topic of the Constitution». E-journal of Portuguese History, 14(2), 51-68. Recuperado de http://www.scielo.mec.pt/pdf/ejph/v14n2/v14n2a03.pdf [12/10/2019]

CARVALHO, S. G. S (2012). Nationalism and regime overthrow in early twentieth century Portugal. Recuperado de http://etheses.lse.ac.uk/404/1/Carvalho_Nationalism\%20and\%20Regime\%20Overthrow\%20i n\%20Early\%20Twentieth\%20Century\%20Portugal.pdf [01/10/2019].

COSTA, S. G. (2008). Azores. Nine Islands, One History. Berkeley: Institute of Governmental Studies Press.

CORDEIRO, C. (2001). «Nos bastidores da visita régia. Decadentismo e tensões autonomistas». Insulana, 57, pp. 5-18.

CORDEIRO, C. (1992). «Autonomia e identidade nacional. Os Açores na Segunda Metade do Século XIX». Revista de História das Ideias, 14, pp. 263-279. 
EASTWOOD, J. (2006). The rise of nationalism in Venezuela. Gainesville, USA: University Press of Florida.

ESTEVE, J. (2017). «La contrarrevolución ibérica. De la implantación del liberalismo al Salazarismo y el Franquismo». En C. RINA SIMÓN (Ed.), Procesos de Nacionalización e Identidades en la Península Ibérica. Cáceres: Universidad de Extremadura, pp. 273-290.

GARÍ, D. (1992). Historia del Nacionalismo Canario. La Laguna: Editorial Benchomo.

GONZÁLEZ, N. (1998). "Crisis de fin de siglo y anglofobia en Canarias». Cuadernos Ateneo de La Laguna, 4, pp. 10-12.

GREENFELD, L. (2018). «The World Nationalism Made». American Affairs, 2(4), pp. 145-59.

Recuperado de https://americanaffairsjournal.org/2018/11/the-world-nationalism-made/ [01/10/2019].

GREENFELD, L. (1992). Nationalism. Five Roads to Modernity. Cambridge, USA: Harvard University Press.

HELBLING, M. (2009). «Nationalism and Democracy: Competing or Complementary Logics?» Living Reviews in Democracy, 1, pp. 1-14. Recuperado de https://ethz.ch/content/dam/ethz/special-interest/gess/cis/cisdam/CIS_DAM_2015/WorkingPapers/Living_Reviews_Democracy/Helbling_updated.pdf [01/10/2019].

HERNÁNDEZ BRAVO DE LAGUNA, J. (2004). «El Nacionalismo y el Regionalismo canarios en torno al siglo XX». Cuadernos del Ateneo, 18, pp. 13-24.

HERNÁNDEZ GONZÁLEZ, M. (2006). Canarias Libre. Secundino Delgado Rodríguez. Biografia y estudio crítico. Santa Cruz de Tenerife: Ediciones Idea.

HERNÁNDEZ GONZÁLEZ, M. (1998). «El nacionalismo canario ante el 98». Cuadernos del Ateneo, 4, pp. 6-9.

HOBSBAWM, E.J. (1992). Nations and Nationalism since 1780. Cambridge, UK: Cambridge University Press

MURO, D. y QUIROGA, A. (2005). «Spanish nationalism. Ethnic or civic? » Ethnicities, 5(1), pp. 9-29.

NEVES, J. (2006). «On Communism and the Nation- Notes from the History of the Colonial Question in the Portuguese Communist Party».

E-journal of Portuguese History, 4(1), 1-11. Recuperado de

https://www.brown.edu/Departments/Portuguese_Brazilian_Studies/ejph/html/issue7/ pdf/jneves.pdf [12/10/2019]

NODIA, G. (1992). «Nationalism and Democracy». Journal of Democracy, 3(4), 3-22.

PAZ SÁNCHEZ, M. (1991). Wangüemert y Cuba (Tomo 1). Santa Cruz de Tenerife: Centro de la Cultura Popular Canaria.

PEREIRA, M. H. (2013). «Crown, Empire and Nation (1807-1834)». E-journal of Portuguese History, 11(1), PP. 43-60.

Recuperado de http://www.scielo.mec.pt/pdf/ejph/v11n1/v11n1a02.pdf [12/10/2019]

PÉREZ DÍAZ, P. (2017). Autonomía insular y regional. La Palma: Cartas Diferentes Ediciones.

POLANAH, P. S. (2011). "“The Zenith of our National History!". National identity, colonial empire, and the promotion of the Portuguese Discoveries: Portugal 1930s». Ejournal of Portuguese History, 9(1), 39-59. Recuperado de https://www.brown.edu/Departments/Portuguese_Brazilian_Studies/ejph/html/issuel 7/pdf/v9n1a03.pdf [12/10/2019] 